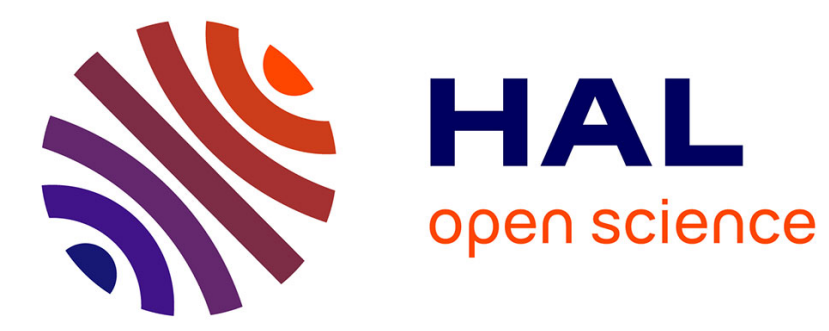

\title{
Passive drag control of a blunt trailing edge cylinder
}

Benjamin Thiria, Olivier Cadot, Jean-François Beaudoin

\section{To cite this version:}

Benjamin Thiria, Olivier Cadot, Jean-François Beaudoin. Passive drag control of a blunt trailing edge cylinder. Journal of Fluids and Structures, 2009, 25, pp.766-776. 10.1016/j.jfluidstructs.2008.07.008 . hal-01289954

\section{HAL Id: hal-01289954 \\ https://hal-ensta-paris.archives-ouvertes.fr/hal-01289954}

Submitted on 24 Nov 2017

HAL is a multi-disciplinary open access archive for the deposit and dissemination of scientific research documents, whether they are published or not. The documents may come from teaching and research institutions in France or abroad, or from public or private research centers.
L'archive ouverte pluridisciplinaire HAL, est destinée au dépôt et à la diffusion de documents scientifiques de niveau recherche, publiés ou non, émanant des établissements d'enseignement et de recherche français ou étrangers, des laboratoires publics ou privés. 


\title{
Passive drag control of a blunt trailing edge cylinder
}

\author{
B. Thiria ${ }^{\mathrm{a}}$, O. Cadot ${ }^{\mathrm{a}, *}$, J.-F. Beaudoin ${ }^{\mathrm{b}}$ \\ ${ }^{a}$ Unité de Mécanique, Ecole Nationale Supérieure de Techniques Avancées, Chemin de la Hunière, 91761 Palaiseau Cedex, France \\ ${ }^{\mathrm{b}}$ Department of Research and Innovation, PSA Peugeot-Citroën, 2 route de Gisy, 78943 Vélizy-Villacoublay, France
}

The turbulent drag of a 2-D bluff body with a blunt trailing edge is investigated for Reynolds numbers in the range $5 \times 10^{3}<\operatorname{Re}<5 \times 10^{4}$. The study focusses on the wake when the drag is reduced by $17.5 \%$ by means of a smaller cylinder located behind the bluff body. Mean pressure and PIV measurements are investigated in the wake. It is shown that the bubble recirculation length and the pressure inside are increased by the presence of the control cylinder. The turbulent wake is also found to have less diffusion compared to that of the natural wake. The global Bénard/von Kármán instability and the corresponding large-scale coherent structures in the far wake are investigated. The global mode envelope of the instability, measured with a hot wire anemometer, is found to be strongly inhibited by the presence of the control cylinder. The maximum amplitudes of the envelope are shifted further downstream the recirculation bubble end and the Strouhal number is slightly increased by $4.3 \%$. Phase averaging of the vorticity field shows that the circulation of the large-scale structures is not significantly affected. A plausible mechanism for the drag reduction is described.

Keywords: Drag; Turbulent wake; Passive control; Global instability

\section{Introduction}

Passive flow control (Gad-el-Hak, 2000) for aerodynamic drag reduction is of great interest for many industrial applications. Among the different methods, there is a simple one (Sreenivasan and Strykowski, 1990; Sakamoto et al., 1991; Sakamoto and Haniu, 1994; Mittal and Raghuvanshi, 2001; Dalton et al., 2001) that consists in using a small secondary body placed behind the main body whose drag has to be reduced. The seminal work of Sreenivasan and Strykowski (1990) concerns the flow of a circular cylinder (the main body) controlled by a secondary smaller cylinder. For this study, the flow Reynolds number is around the critical value for vortex shedding. For certain locations of the secondary cylinder, the authors find a wake stabilization that could go towards the complete shedding suppression and the drag reduced by $20 \%$. They propose a stabilization mechanism based on the modification of interaction of the shear layers in the frame of the model of Gerrard (1966). Mittal (2001) and Mittal and Raghuvanshi (2001) investigated numerically the same flow at $\mathrm{Re}=100$. The computation is in fairly good agreement with the Sreenivasan and Strykowsky results. They argued for another mechanism, related to the favourable pressure gradient locally introduced

\footnotetext{
*Corresponding author.

E-mail address: olivier.cadot@ensta.fr (O. Cadot).
} 
by the control cylinder that might stabilize one of the shear layer. The consequences of this passive control on the drag and the lift oscillations have been studied numerically and experimentally by Dalton et al. (2001), also for larger but still moderate Reynolds numbers from 100 to 3000. They mainly find that in addition to the mean drag decrease, the oscillations of drag and lift are reduced by the presence of the secondary cylinder. Moreover, the asymmetry introduced by the control cylinder produces a small nonzero mean lift component on the overall system.

For large Reynolds numbers, the method is also very successful in reducing forces. Sakamoto et al. (1991) and Sakamoto and Haniu (1994) investigated in detail the method applied to square and circular primary cylinders for $\operatorname{Re}>10000$. For certain positions of the control cylinder, the drag can be reduced by up to $40 \%$ for the circular cylinder and $30 \%$ for the square cylinder. The difference in magnitude is essentially due to the supplementary drag reduction raised by the separation point displacements that are only possible for the circular cylinder. When the separation points are fixed, which is the case for the square cylinder, the maximum reduction occurs when the control cylinder is located near the outer boundary of the separated shear layer in the vicinity of the bluff body. This position at large Re is very similar to the position for shedding suppression observed at low Reynolds number by Sreenivasan and Strykowski (1990).

These effects recall previous studies concerning bluff body wakes with splitter plates (Apelt and West, 1975). The splitter plate placed on the symmetry axis at the rear of the main body inhibits the coupling of the opposite shear layers. Even for small splitter plates, the base pressure is drastically increased and consequently the drag reduced. These effects are associated to a larger recirculation bubble and a reduction of the shedding frequency. These observations was used by Gerrard (1966) to illustrate a plausible interaction mechanism for vortex shedding. It is worth noticing that flow configurations implying two bluff-bodies, or more, belong now to a wide research field dealing with aerodynamic wakes interferences (Zdravkovich, 1987; Mahbub Alam and Zhou, 2008).

The aim of our work is to give more insight into the physical mechanisms involved in the passive drag reduction method using a smaller control cylinder. It is also a contribution for the puzzle of the comprehension of the drag origin of 2-D bluff-bodies wakes. Actually, the relationship of global flow quantities, as the shedding frequency and the strength of the shed eddies with the mean base pressure still remains unclear (Roshko, 1993). The fundamental question for form drag is: what governs the pressure level in the recirculation bubble, especially when the wake is unsteady? For passive control using a smaller cylinder, there are no studies which investigate the wake dynamics at large Reynolds number. A question is whether the flow modification introduced by a small control cylinder in the wake is similar to that of splitter-plate control. We then propose to measure some properties of the global dynamics of the wake when the drag is reduced for Reynolds numbers in the range $5 \times 10^{3}<\operatorname{Re}<5 \times 10^{4}$.

The article is organized as follows. We first describe in Section 2 the experimental set-up. The results presented in Section 3 concerns the mean wake modifications (Section 3.1) and the global dynamics modification (Section 3.2). The stabilization mechanism of the global instability and the drag reduction are discussed in Section 4. We conclude in Section 5 .

\section{Experimental set-up and measurements}

The Eiffel type wind tunnel is an open loop air-flow facility. The turbulent intensity is less than $0.3 \%$ and the homogeneity of the velocity over the $400 \mathrm{~mm} \times 400 \mathrm{~mm}$ blowing section is $\pm 0.4 \%$. The wake is produced by a symmetric cylinder (that we will later refer to as the primary cylinder) with a leading face profiled as a semi-circle, and a flat trailing plane at right angles to the flow. The width of the trailing vertical plane is $D=25 \mathrm{~mm}$ (arrangement and dimensions are depicted in Fig. 1). The wind velocity is measured with a Pitot tube located upstream from the model. The Reynolds number of the primary cylinder is defined as $\mathrm{Re}=U D / v$. We investigated the wake in the range $5 \times 10^{3}<\operatorname{Re}<5 \times 10^{4}$. The control cylinder has a diameter of $d=3 \mathrm{~mm}(d / D=0.12)$. It is directly fixed on the bluff blunt body. For drag measurements, the bluff bunt body is fixed by one of its sides to an aerodynamic balance giving the two components of a static force, say the drag and lift. The sensitivity of the balance is $0.31 \mathrm{~V} / \mathrm{N}$ for the drag and $0.15 \mathrm{~V} / \mathrm{N}$ for the lift. The wind blows the model uniformly over a length of $400 \mathrm{~mm}$, while the total span of the model is $570 \mathrm{~mm}$. On each side of the uniform blowing length, the velocity decreases to zero on a typical length of $15 \mathrm{~mm}$. The value of drag is certainly biased by the end effects, but the drag reduction observed between the controlled and the uncontrolled cases is comparable to other studies.

A hot-wire probe from DANTEC is used for the measurements of local velocity fluctuations. The wire is oriented in such a way to be sensitive to the modulus of the velocity in the plane $x O y$. The hot wire is replaced by a thin Pitot tube $(3 \mathrm{~mm}$ in diameter) for local pressure measurements. Only its static pressure hole is connected to the differential pressure manometer (from Validyne). The other input of the manometer is connected to the static pressure of the main 


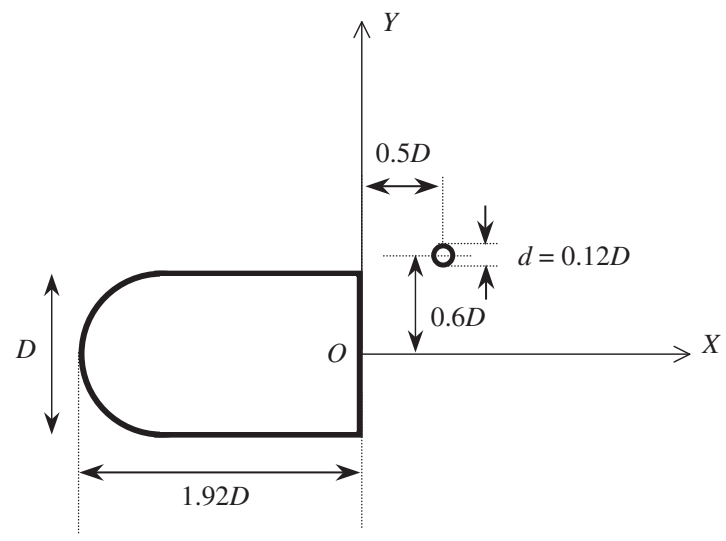

Fig. 1. Dimensions of the bluff body $(D=25 \mathrm{~mm})$ and small control cylinder arrangement.

Table 1

Drag measurements between the overall system (blunt body + control cylinder) and the blunt body only (DR means drag reduction).

\begin{tabular}{lllll}
\hline$U(\mathrm{~m} / \mathrm{s})$ & 12 & 16 & 20 & 24 \\
$\operatorname{DR}(\%)$ & $17.4 \pm 5$ & $16.9 \pm 2.8$ & $17.5 \pm 1.8$ & $17.5 \pm 1.3$ \\
\hline
\end{tabular}

flow upstream the cylinder. The measurements points, either velocity or pressure, are all located in the plane $x O y$ of Fig. 1. The main cylinder is also equipped with 16 pressure taps in its central section connected to a Scanivalve device of type DSA 3217/16px.

The flow structure is investigated through visualizations and PIV measurements in the plane $x O y$ of Fig. 1. Both are achieved using a LAVISION particle image velocity set-up. The light sheet is produced by two Nd:Yag lasers of $50 \mathrm{~mJ}$ per pulse. The seeding is insured by an olive-oil spray placed at the entrance of the wind tunnel. For PIV intercorrelation measurements where homogeneous seeding is needed, the entire room, containing the wind tunnel facility, is seeded before PIV acquisitions are performed.

\section{Results}

We checked that the overall drag has effectively been reduced. The balance measures the drag of both the blunt body and the control cylinder. Table 1 gives the values of this total drag reduction for different wind velocities. The drag reduction magnitudes are about $17.5 \%$. We observe a small positive mean lift component creation (in the direction of positive $y$ in Fig. 1) of about 4\% the total drag of the cylinder without control.

\subsection{Mean flow modification}

The presence of the control cylinder does not modify the flow around the forehead body as displayed by the visualization in Fig. 2. In each case, the detachment does not occur exactly at right angles to the blunt trailing edge, but upstream, after the junction between the profiled forehead body and the horizontal side. On the other hand, the presence of the control cylinder strongly modifies the wake. In Fig. 3(a), the natural wake is dominated by the shedding of large vortical structures. We can see in Fig. 3(b) that the control cylinder delays the shedding of these large vortical structures and produces its own wake at a higher frequency.

The pressure distribution around the main body presents the same characteristics as the visualization. Fig. 4 shows the pressure coefficient $C_{p}$ defined as

$$
C_{p}=\frac{p-p_{0}}{p_{s}-p_{0}}
$$



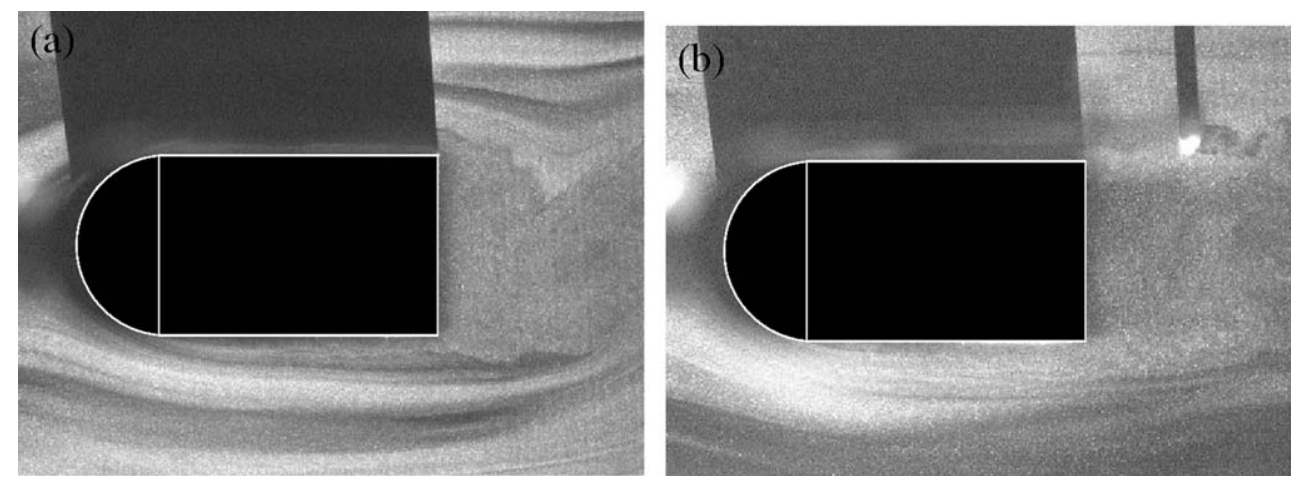

Fig. 2. Flow visualization around the body: (a) natural flow and (b) with the control cylinder.
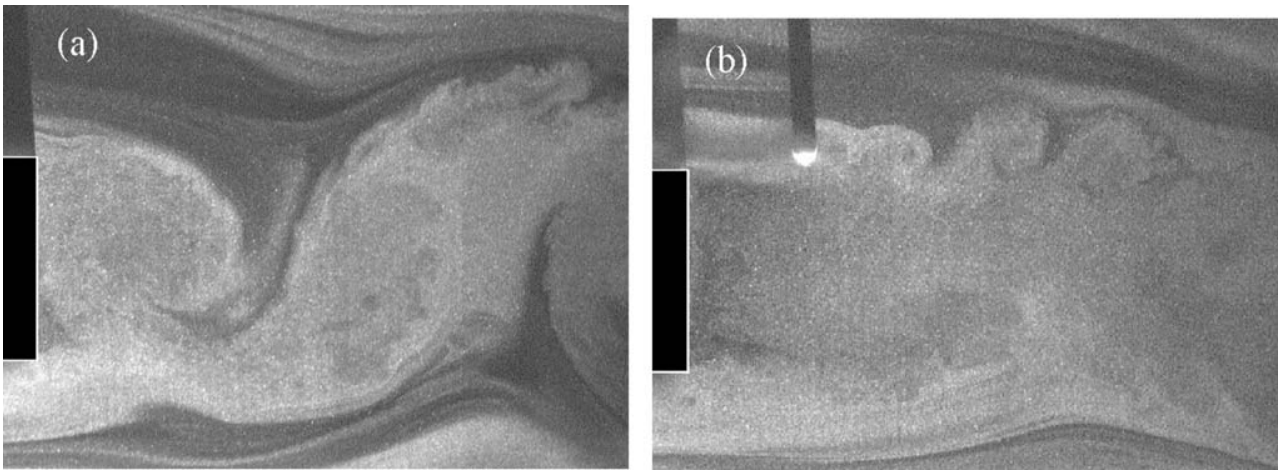

Fig. 3. Wake visualization: (a) natural wake and (b) with the control cylinder.

where $p$ is the static pressure, $p_{0}$ the free-stream pressure and $p_{s}$ the pressure at the mean stagnation point on the forehead body. The $C_{p}$ distribution remains unchanged all over the forehead body. At the rear of the body, the base pressure coefficient is significantly increased from -0.57 for the natural wake to -0.31 for the controlled case. As for large Reynolds numbers, the exerted drag is directly associated to the pressure distribution around the body (neglecting viscous stresses), the base pressure increase is then the cause for the observed drag reduction. The small component of lift that was measured with the aerodynamic balance is hardly discernible since the figure is rather symmetric. However, over parts 4 and 5 (lower part of the body), the pressure with control is slightly increased compared to that of parts 1 and 2 (upper part of the body). This is consistent with the small positive lift creation. The local mean pressure in the wake is displayed in Fig. 5. For the natural wake in Fig. 5(a), the minimum of the pressure coefficient is -0.76 at $x / D=0.25, y / D=0$. The base pressure given in Fig. 4 is then not the minimum of the pressure since there is a significant difference of 0.2 between them. This observation is consistent with the numerical computations in Balachandar et al. (1997) who obtained a difference of a similar value for a circular cylinder and a normal plate. Our intrusive local pressure measurements seem reliable. For the controlled wake, we did not measure the pressure for distances smaller than $x / D<0.7$ because the displacement of the Pitot tube is not realizable. Nevertheless, the pressure in the controlled wake is dramatically modified as shown in Fig. 5(b) even farther downstream from the body. In the measured region, the minimum of the pressure coefficient is -0.33 which is again lower than the base pressure given in Fig. 4. All the pressure levels are strongly increased compared to those of the natural wake. This effect extends over distances much larger than $D$ around the rear of the body.

A main characteristic of separated flows is the size of the recirculation region. The streamlines of the mean flow in Fig. 6 obtained with the PIV technique exhibits the recirculation region for both wakes. The recirculation length is defined as the streamwise distance starting from the rear of the body at $x / D=0$ to the stagnation point in the wake. The length increases from about $0.9 D$ for the natural wake to approximately $1.25 D$ with the control cylinder. Magnitudes of the mean longitudinal velocity ( $x$-component) are compared for both cases in Fig. 7(a). The wake of the control cylinder is clearly distinguishable at the top of the third velocity profile (from the left in Fig. 7(a)) and disappears after $x / D>1.8$. The main observation in this figure is that the lower velocities (observed on the central line 


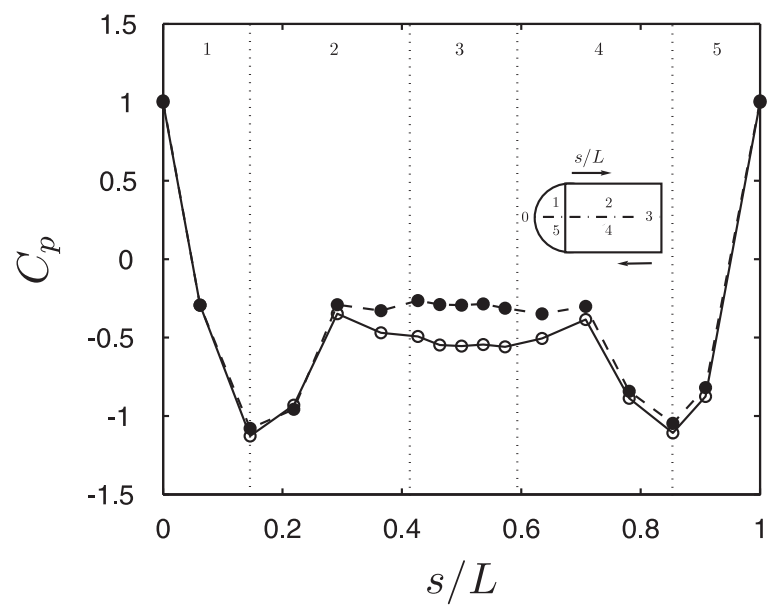

Fig. 4. Pressure distribution around the natural blunt body (०) and the blunt body with the control cylinder (•).
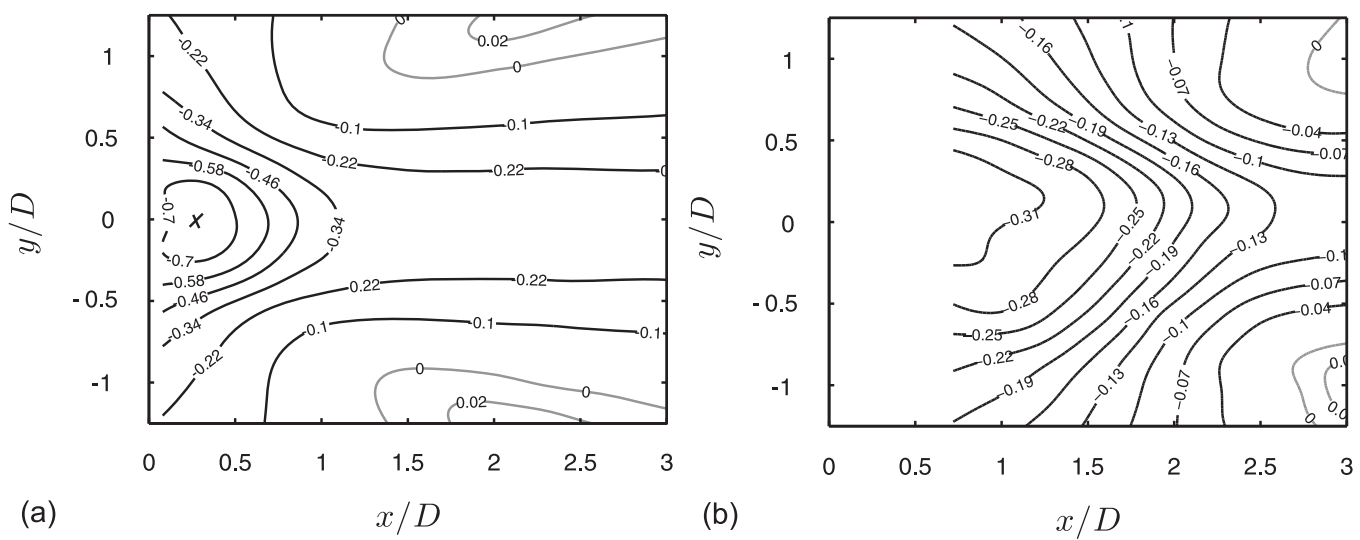

Fig. 5. Isocontour of the static pressure at $U=22 \mathrm{~m} / \mathrm{s}$ : (a) natural wake, the cross indicates the global minimum -0.76 ; (b) controlled wake.

of the wake) relax to the free-stream velocity much slower for the controlled wake than for the natural one. Consequently, the mean gradient is larger in the controlled wake, indicating a reduction of the eddy viscosity compared to that of the natural wake. Fig. 7(b) shows the square root of the mean enstrophy denoted by $\Omega$ and defined as

$$
\Omega=\sqrt{\left\langle\omega_{z}^{2}\right\rangle},
$$

where $\omega_{z}=\partial_{x} v-\partial_{y} u$ is the $z$-component of the vorticity and where \langle\rangle denotes a time averaging. The enstrophy is produced in the boundary layers of the body. For the natural wake, the enstrophy is initially symmetrically distributed around two maxima corresponding to the shears at $(x / D=0 ; y / D= \pm 0.5)$ in Fig. 7(b). Downstream, the enstrophy is quickly mixed and diffused. As a result, only one maximum is present in the profiles for $x / D>0.8$. The mixing occurs around the end of the recirculation region at $0.9 D$ corresponding to the third velocity profile in Fig. 7(b). For the controlled case, the two maxima are present on a much longer distance in the wake. They disappear into a single maximum only after $x / D>1.8$ which is further downstream the end of the recirculation region at $1.25 D$ corresponding to the fourth velocity profile in Fig. 7(b). This confirms the reduction of turbulent diffusion produced by the control cylinder. We also observe that the total enstrophy along vertical profiles is qualitatively comparable for both wakes; its production is then not significantly modified by the addition of the small control cylinder.

In conclusion, the mean flow properties modification indicates that the drag reduction is due to a global large pressure increase in the wake. It is accompanied with an increase in the recirculation length, a delay of the "mixing" 

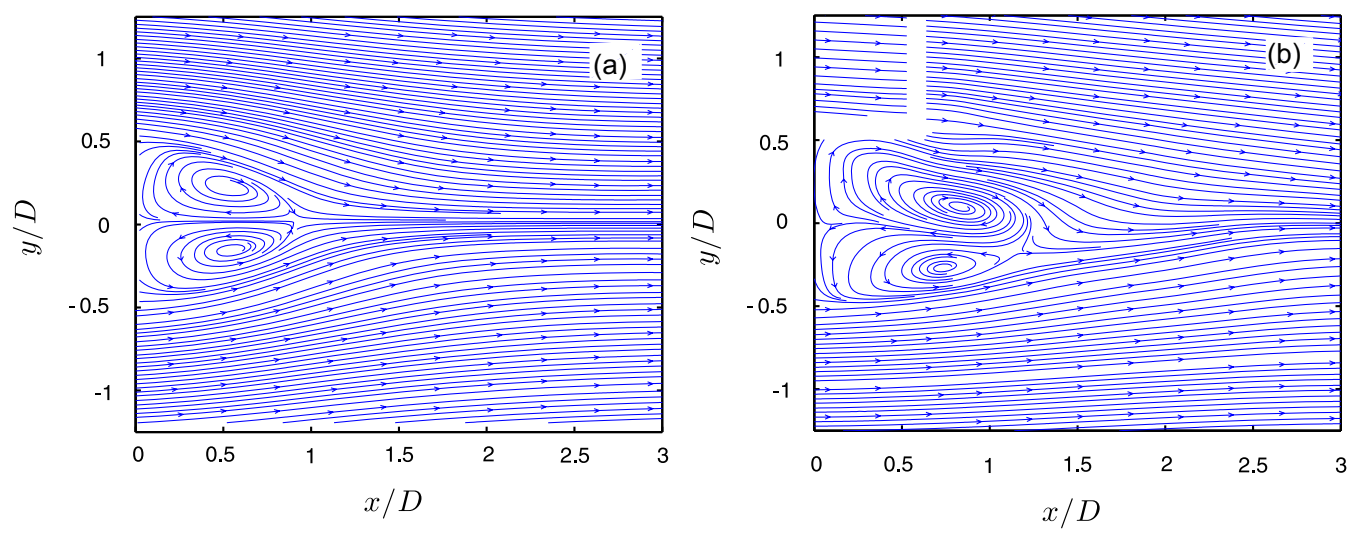

Fig. 6. Streamlines of the mean flow computed from 1000 instantaneous velocity fields at $U=22 \mathrm{~m} / \mathrm{s}$ : (a) natural wake, (b) controlled wake.
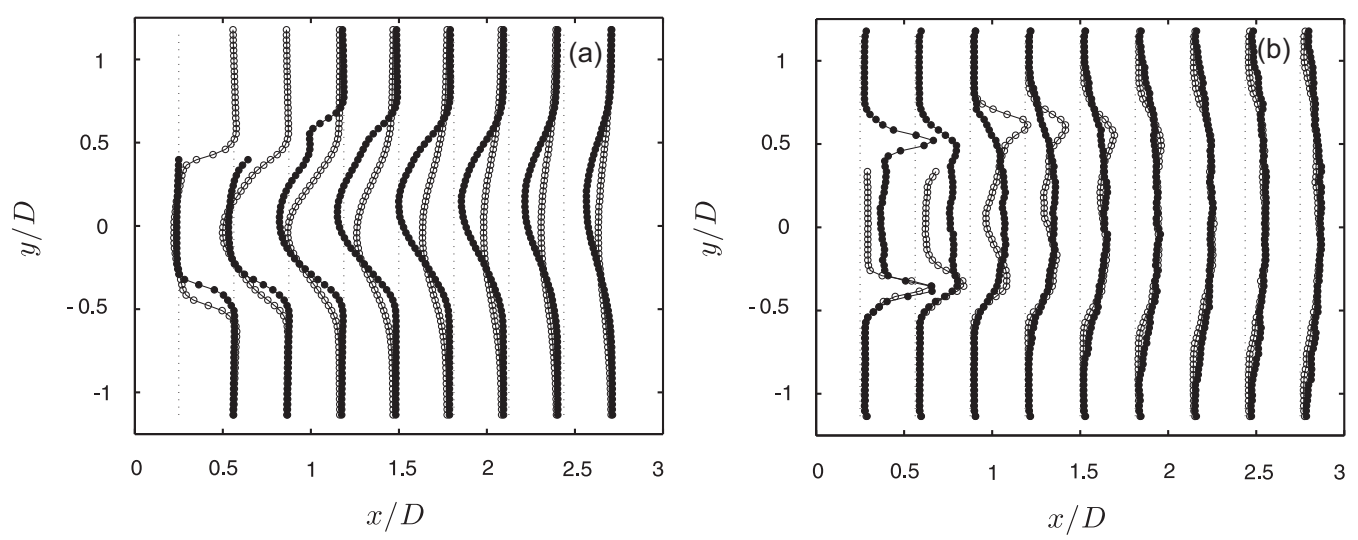

Fig. 7. Profiles of the longitudinal component of the mean velocity fields: (a) profiles of the square root of the mean enstrophy, (b) for the controlled (empty circles) and natural (filled circles) wakes at $U=22 \mathrm{~m} / \mathrm{s}$.

corresponding to a less diffused wake. In the next section we study the modification of the global dynamics of the wakes.

\subsection{Global wake dynamics modification}

The rate of acquisition of our PIV system is too slow $(15 \mathrm{~Hz})$ to capture the dynamics of the turbulent wake. Hence, we then performed hot-wire measurements in order to extract the velocity fluctuations spectrum for different locations in the wake. The local velocity spectrum in 2-D bluff body wakes is dominated by the global synchronization of the flow on a single frequency $f_{0}$ (i.e., the shedding frequency). The amplitude of the peak at the synchronization frequency measured in space, say $A_{f_{0}}(x, y)$, defines the so called global mode envelope (Zielinska and Wesfreid, 1995). This envelope is characteristic to the underlying global instability (Chomaz, 2005). We show, in Fig. 8, two spectra measured at a point in the wake where the amplitude of the global mode is maximum. Each peak in Fig. 8 corresponds to the global mode. The synchronization frequency is slightly increased with the control cylinder. For the natural, the global frequency is $211 \mathrm{~Hz}$ corresponding to a Strouhal number of $\mathrm{St}=f d / U=0.238$. For the controlled wake, the frequency is $220 \mathrm{~Hz}$ corresponding to a Strouhal number of $\mathrm{St}=0.248$. At each location in the wake, $A_{f_{0}}(x, y)$ is extracted and plotted on the contour map presented in Fig. 9(a) and (b). For the natural wake, the envelope is symmetric, as expected by the experimental symmetry. Two maxima of magnitudes about $2.86 \mathrm{~m} / \mathrm{s}$ are found very close to the rear of the bluff body at $x / D=0.6 D$ and symmetrically located at $y / D= \pm 0.4 D$. For the controlled wake, the maxima are significantly damped by a factor of 2 (see also Fig. 8); their magnitudes are now about $1.36 \mathrm{~m} / \mathrm{s}$ and located much further 


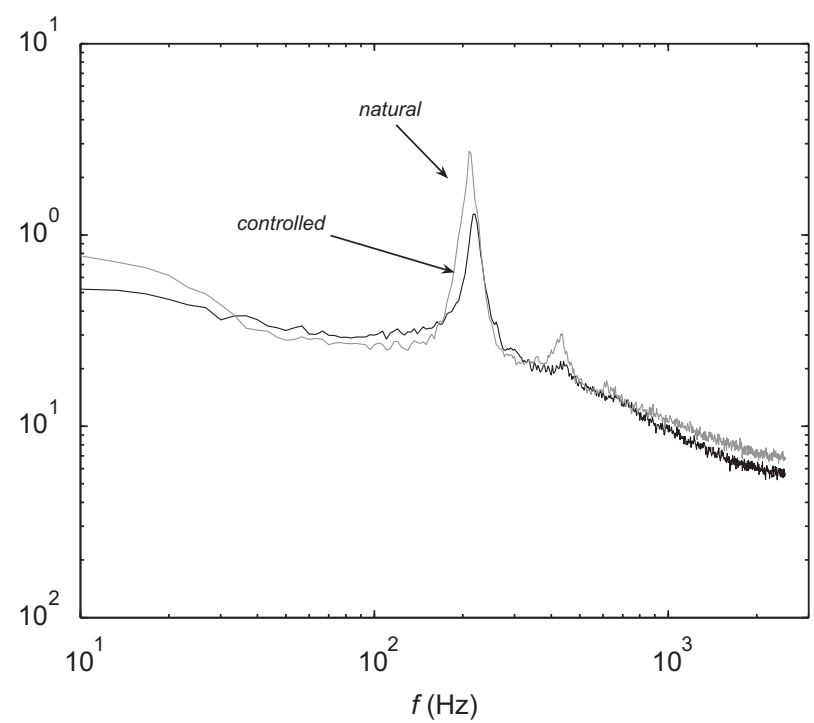

Fig. 8. Amplitude (rms) spectra of the velocity modulus fluctuations in $\mathrm{m} / \mathrm{s}$ for natural and controlled case at $U=22 \mathrm{~m} / \mathrm{s}$. They are measured at the location where the peak amplitude is maximum in the wake (see Fig. 9(a) and (b)). The frequency at the peak location is the global frequency of the wake.
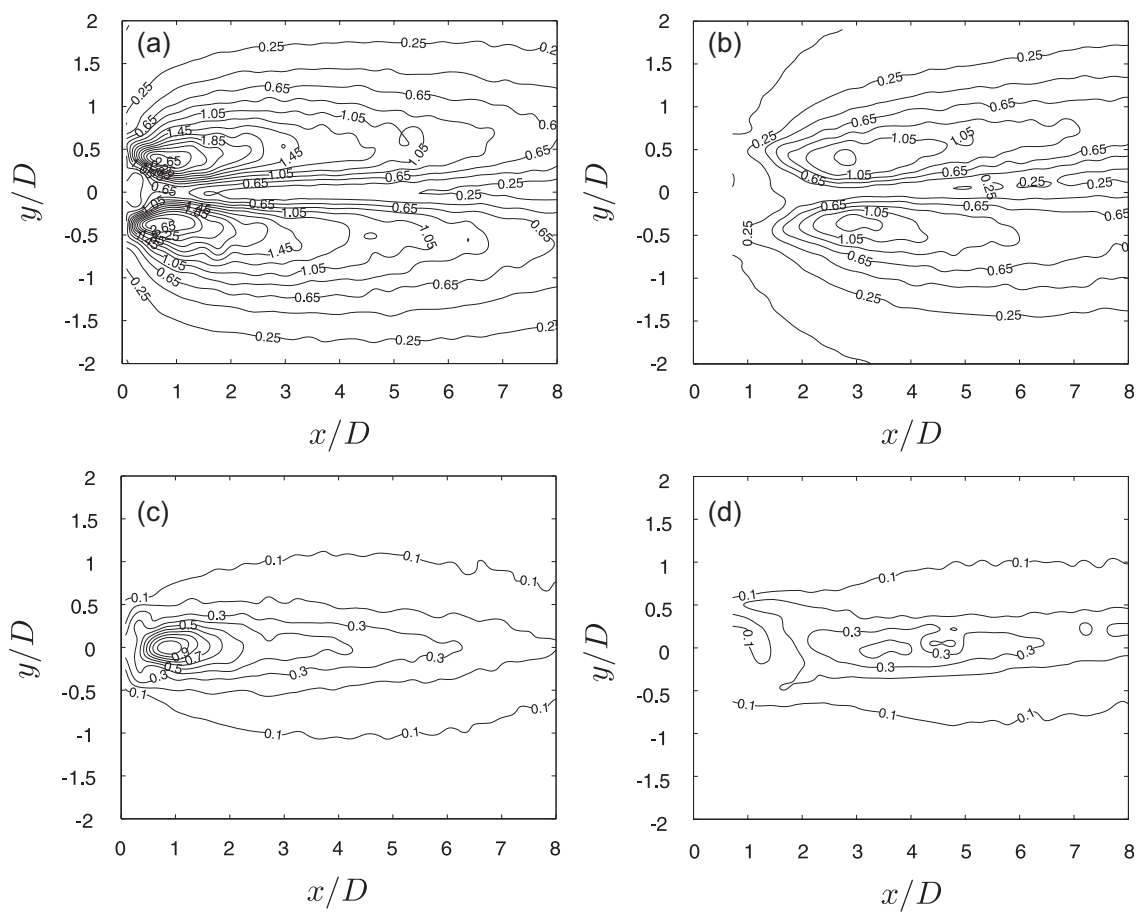

Fig. 9. Global mode envelopes in $\mathrm{m} / \mathrm{s}$. Envelope for the fundamental synchronization frequency $A_{f_{0}}(x, y)$ for (a) the natural wake and (b) the controlled wake. Envelope for the first harmonic $A_{2 f_{0}}(x, y)$ for (c) the natural wake and (d) the controlled wake.

downstream in the wake at $x / D=2.8$. The positions are still rather symmetrically located as for the natural wake at positions $y / D= \pm 0.4 D$, while the model configuration is now not symmetric anymore. As displayed in Fig. 9(c) and (d), the envelope at the first harmonic, $2 f_{0}$ is also damped and shifted downstream by the presence of the control cylinder. 
We show two contour maps of the instantaneous vorticity $\omega_{z}$ for both wakes. In Fig. 10(a), we can easily recognize the formation of the large vortical structures shown by the visualization in Fig. 3(a). In Fig. 10(a), we can see the upper sheet of negative vorticity rolling up into an eddy, another eddy of opposite vorticity sign in the middle of the figure which has been shed from the lower sheet, and further downstream a last eddy of negative vorticity shed from the upper sheet. The separation distance between two consecutive eddies of the same vorticity sign is marked as $\Lambda$ in the figure. With the control cylinder, the upper sheet rolls up into several eddies of the same vorticity sign having a separation distance of $\lambda \approx 0.4 D$, while the lower sheet rolls up into a single vorticity patch as in the natural case.

Although the PIV system is slow, we can still obtain a phase-averaged velocity field. It is very useful in order to capture the large-scale dynamics of the flow. What we really need is to average the flow at a certain phase of the global dynamics defined from its synchronization frequency. However, the fact that the wake is synchronized is not a sufficient condition to catch a fixed phase. The turbulent wake often dislocates under 3-D disturbances and produces phase jumps in the wake. In order to not lose the phase information, we modified the apparatus. The primary cylinder is attached by two flexible rods allowing a small vertical displacement. An accelerometer, fixed to one edge of the cylinder, gives the phase of the displacement. The stiffness of the rods and the mass of the body define the natural oscillation frequency of the structure, which is $30 \mathrm{~Hz}$. When the aerodynamic excitation frequency (the lift) is comparable to the structure natural frequency, there is a lock-in configuration where the oscillations are phase-coherent to the lift. Technically, the PIV is triggered by the accelerometer at a certain phase. The same experiment is performed for an identical phase of the acceleration with and without the control cylinder. The vertical displacement amplitude never exceeds $1 \%$ of the bluff body size $D$. However, the flow is probably now more "2-D" than "3-D" due to the small but coherent spanwise forcing
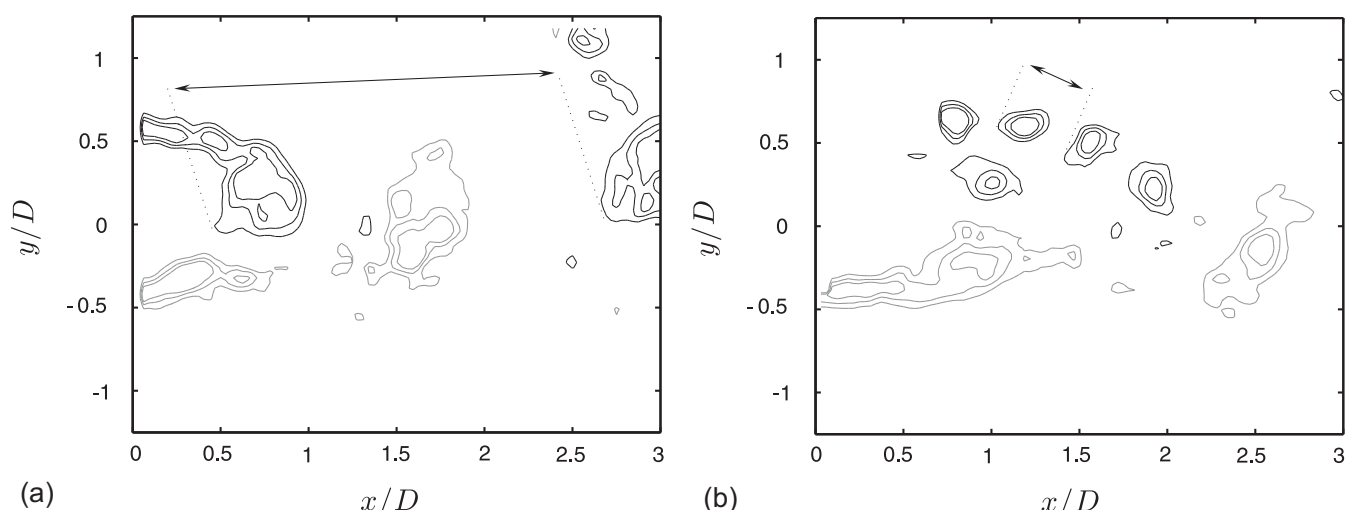

Fig. 10. Instantaneous vorticity $\omega_{z}$ fields at $U=22 \mathrm{~m} / \mathrm{s}$. Black contours correspond to negative vorticity (clockwise rotation) and grey contours to positive vorticity (anticlockwise rotation). The isovalues are $\pm 4000, \pm 3000$ and $\pm 2000 \mathrm{~s}^{-1}$. (a) Natural wake and (b) controlled wake.
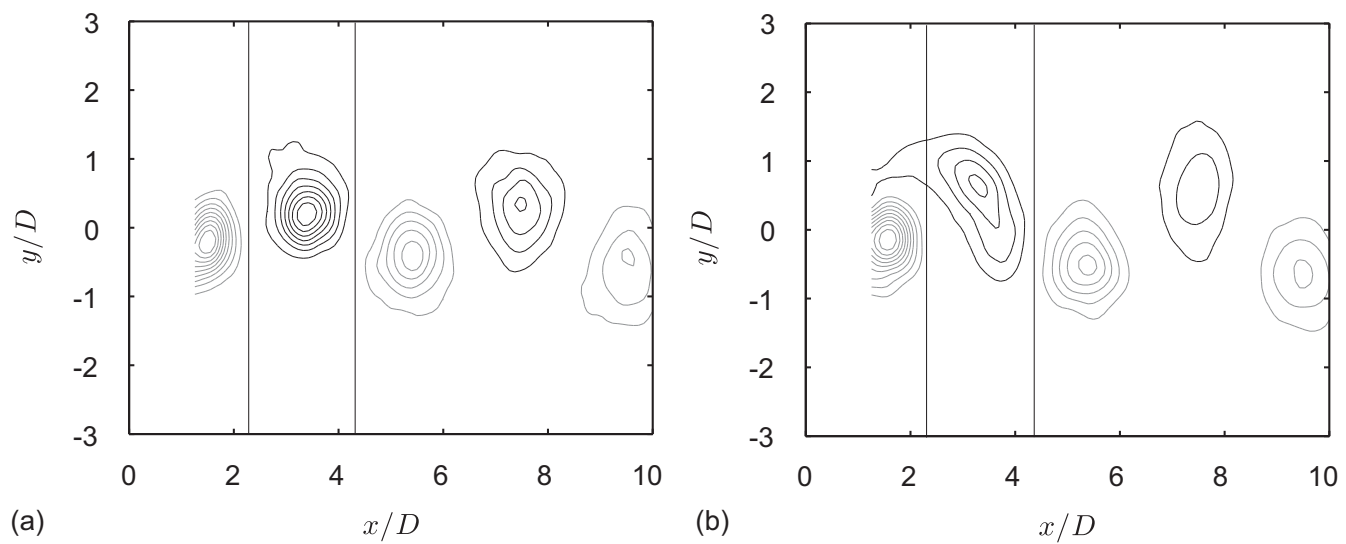

Fig. 11. Phase average of the vorticity performs on the Kármán frequency at $U=3 \mathrm{~m} / \mathrm{s}$. Black contours correspond to negative vorticity (clockwise direction) and grey contours to positive vorticity. (a) Natural wake, (b) controlled wake. Vertical lines limit the area on which the circulation plotted in Fig. 12 is computed. 


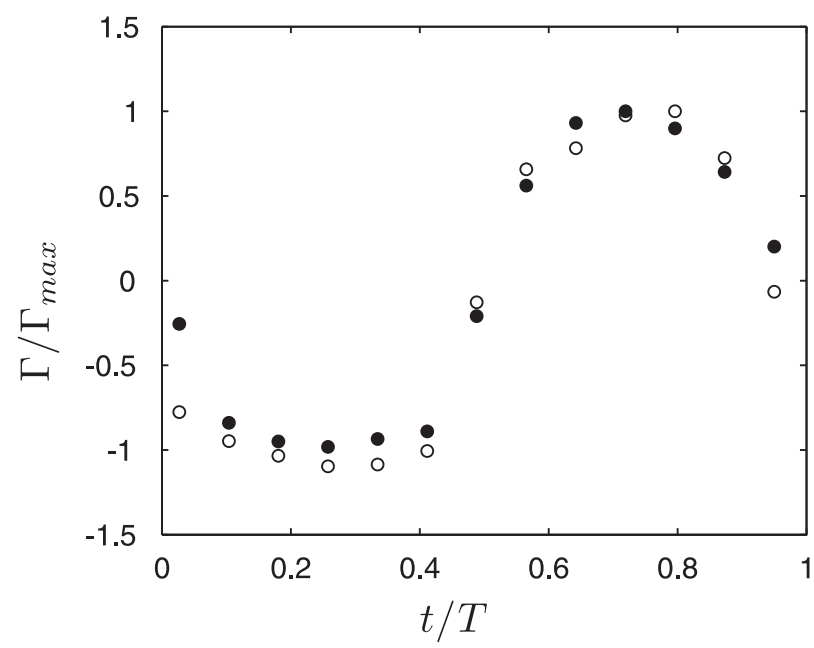

Fig. 12. Circulation of the time-dependent mean flow computed over areas displayed in Fig. 11 for the controlled wake (o) and the natural wake (•). The circulation is divided by the maximum value $\Gamma_{\max }$ obtained for the natural wake.

created by the vibrations all along the cylinder span [see, for example, Poncet, 2004, in the case of a rotating circular cylinder]. We checked that the base pressure was not modified by the vibration, which means that the drag reduction due to the secondary cylinder is still present even with the vibration and the phase lock-in.

The synchronization with the natural frequency of $30 \mathrm{~Hz}$ can be performed for $U=3 \mathrm{~m} / \mathrm{s}$. The synchronization is checked using the visualization technique. The flow is averaged over 200 velocity fields. Fig. 11(a) and (b), respectively, show the contours of iso-vorticity without and with the control cylinder at the same phase of the lift excitation. At this frequency, the mean flow is similar to the laminar Kármán street observed at lower Reynolds number. The presence of the control cylinder affects considerably the formation of the vorticity structure, but only on the side of the control cylinder location. Effectively, the vorticity patch is not circular as in the case of the natural wake (Fig. 11(a)), but is significantly flattened and the maximum of the vorticity reduced. The flattened eddy is the result of the incoherent average of the smaller eddies shown in Fig. 10(b) and produced by the control cylinder.

We perform phase averaging for a few time delays with the initial trigger and obtain 13 mean flows equally phaseseparated in a cycle of lift oscillation. We then construct a time-dependent mean flow over the period $T$. We extract from this time-dependent mean flow the circulation computed over an area having half the Kármán street wavelength in width and the size of the PIV measurements domain in height (this area is displayed in Fig. 11). This area is periodically crossed by mean vorticity structures. The circulation is maximum with positive magnitude when the area contains totally a mean vorticity patch from the bottom street. Respectively, the circulation is minimum with negative magnitude when the area contains totally a mean vorticity patch from the top street (which is the case for Fig. 11). The circulation oscillation is shown in Fig. 12 for the natural and the controlled wake. Both circulations are very similar in magnitude. Hence, the reduction of the maximum of the vorticity contained in the flattened eddy is compensated by its increased area (related to its elongated shape, see Fig. 11(b)) to keep the circulation constant.

If we look closer at Fig. 12, we can see a small asymmetry between the two curves, showing that, for the controlled case, there is more negative circulation coming from the upper part of the wake (where the control cylinder is) than positive circulation coming from the lower part. This can be related to the small lift measured with the balance and observed in Fig. 4 and created by the asymmetry of the mean controlled flow. It is also related to the fact that the incoming flow is not uniform on the control cylinder (see Fig. 7(a)), that introduces more negative than positive circulation into the flow.

\section{Discussion}

\subsection{Modification of the Bénard von Kármán (BvK) global instability}

The presence of the control cylinder modifies the shape of the global mode envelope of the Bénard/von Kármán instability (see Fig. 9(b) and (d)). The envelope is mainly considerably shifted downstream together with a damping of 
its amplitude. For controlled wakes, or forced wakes in general, Thiria and Wesfreid (2006) have shown that the global downstream shift of the envelope together with the reduction of its maximum, which testifies from a weaker growth rate of disturbances, carries out a stabilization of the instability. The stabilization should then be understood as a delay in the global dynamics of the Bénard/von Kármán instability together with a reduction of the associated velocity fluctuations.

The stabilization is not associated to a drastic change of the shedding frequency. We observe a small but significant increase in the frequency of the vortex shedding, by $4.3 \%$ (see Fig. 8). Similar results were reported before for large Reynolds number experiments by Bearman (1965) using a splitter plate as passive control and Sakamoto et al. (1991) using a control cylinder. Near the threshold of the instability, the shedding frequency is found to be decreased by the presence of the control cylinder, because the primary stabilization effect of the control cylinder is to postpone the onset of vortex shedding to a higher Reynolds number (Sreenivasan and Strykowski, 1990). In Mittal and Raghuvanshi (2001) the Strouhal number is slightly increased by the presence of the control cylinder at $\mathrm{Re}=100$ in accordance with our experiment at a larger Reynolds number. We can interpret the frequency increase in view of the model of Gerrard (1966). In this model of vortex shedding, the thickness of the shear layers at the end of the formation length (characteristic of the length of the recirculation region) is related to the shedding frequency; the thinner the shear layers, the larger the frequency. In our case, the control cylinder reduces the eddy viscosity (results shown in Fig. 7). As a result, the vorticity layers displayed in Fig. 7(b) are much thinner at the end of the recirculation region for the controlled case than for the natural case. Effectively, with the control cylinder the recirculation region ends around $1.25 D$ (Fig. 6(b)) and around $0.9 D$ (Fig. 6(b)) for the natural wake. For the controlled case the vorticity is still distributed around two maxima corresponding to the two shear layers at $1.25 \mathrm{D}$, while for the natural case the shears are not even distinguishable at $0.9 \mathrm{D}$. This effect is also found in the instantaneous vorticity field of Fig. 10. For the controlled case, the upper vorticity layer that is disturbed by the control cylinder keeps its thickness nearly constant until $x / D \approx 1.5$, that is even beyond the size of the recirculation bubble. This contrast might be at the origin of the shedding increase. The massive increase in the recirculation bubble length might be the consequence of the downstream shift of the global mode envelope, and then to the stabilization or the delay of the Bénard/von Kármán instability. We believe that this stabilization takes its origin in the mean flow modification that accentuates the convective nature of the instability

\subsection{Drag reduction}

The drag reduction is directly linked to the increase in the base pressure coefficient (see Fig. 4). This increase is associated to an increase in the recirculation length. It is an evolution that is characteristic to wakes in general and well reproduced by the cavity models $(\mathrm{Wu}, 1978)$. The reason is that the larger the formation length, the less curved the instantaneous flow at the rear of the body, implying a reduction of the pressure gradient due to the centrifugal forces. We can thus propose the following plausible mechanism. When the flow is natural, the Bénard/von Kármán instability is mainly responsible for the bubble closure since the maxima of the envelope are located inside the recirculation bubble. Hence, the Bénard/von Kármán instability and the drag exerted on the body are intimately related. The control cylinder stabilizes the Bénard/von Kármán instability by shifting downstream the global mode envelope. The bubble closure is then delayed downstream. Consequently, the length increase causes the pressure increase in the bubble and then leads to the reduced drag.

\section{Concluding remarks}

In this article, we studied experimentally the passive drag control of a turbulent wake by a small secondary cylinder placed behind a bluff body. For appropriate localization, a maximum drag reduction of $17.5 \%$ can be achieved. The mechanism for drag reduction involves neither moving the separation points nor reduction of the circulation production, nor shedding frequency reduction. The synchronization of the global Bénard/von Kármán instability is unaffected but the global mode envelope is shifted downstream the recirculation bubble closure and its maximum is reduced. The growth rate of the instability is then altered but the selected global frequency remains, at first order unchanged (slightly increased).

This study offers a perspective on the understanding of the stability properties of an open flow governed by an absolute instability at large Reynolds number. The placement of the control cylinder into the flow that modifies the global properties (here the drag) shows the global sensitivity to a local perturbation. 


\section{Acknowledgements}

We would like to thank M. Rossi and J.-M. Chomaz for useful discussions as well as T. Pichon and M. Leriche for their help on the experimental set-up. The work benefited from the financial help of PSA Peugeot-Citroën and the Direction Générale de l'Armement. PSA Peugeot-Citroën supported the Postdoc position of B.Thiria. This work was supported by the ANR project no. 06-BLAN-0363-01 "HighSpeedPIV”.

\section{References}

Apelt, C.J., West, G.S., 1975. The effects od wake splitter plates on bluff body flow in the range $10^{4}<R<5.10^{4}$ Part 2 . Journal of Fluid Mechanics 71, 145-160.

Balachandar, S., Mittal, S., Najjar, F.M., 1997. Properties of the mean recirculation region in the wakes of two-dimensional bluff bodies. Journal of Fluid Mechanics 351, 167-199.

Bearman, P.W., 1965. Investigation of the flow behind a two-dimensional model with a blunt trailing edge and fitted splitter plates. Journal of Fluid Mechanics 21, 241.

Chomaz, J.-M., 2005. Global instabilities in spatially developing flows: non normality and nonlinearity. Annual Review of Fluid Mechanics 37, 357-392.

Dalton, C., Xu, Y., Owen, J.C., 2001. The suppression of lift on a circular cylinder due to vortex shedding at moderate Reynolds numbers. Journal of Fluids and Structures 15, 617-628.

Gad-el-Hak, M., 2000. Flow Control. Cambridge University Press, Cambridge.

Gerrard, J.H., 1966. The mechanics of the formation region of vortices behind bluff bodies. Journal of Fluid Mechanics $25,401-413$.

Mahbub Alam, Md., Zhou, Y., 2008. Strouhal numbers, forces and flow structures around two tandem cylinders of different diameters. Journal of Fluids and Mechanics 24, 505-526.

Mittal, S., 2001. Control of flow past bluff bodies using rotating control cylinders. Journal of Fluids and Structures 15, 291-326.

Mittal, S., Raghuvanshi, A., 2001. Control of vortex shedding behind circular cylinder for flows at low Reynolds numbers. International Journal for Numerical Methods in Fluids 35, 421-447.

Poncet, P., 2004. Topological aspects of three-dimensional wakes behind rotary oscillating cylinders. Journal of Fluid Mechanics 517, 27-53.

Roshko, A., 1993. Perspectives on bluff body aerodynamics. Journal of Wind Engineering and Industrial Aerodynamics 49, 79-100.

Sakamoto, H., Haniu, H., 1994. Optimum suppression of fluid forces acting on circular cylinder. ASME Journal of Fluids Engineering $113,183-189$

Sakamoto, H., Tan, K., Haniu, H., 1991. An optimum suppression of fluid forces by controlling a shear layer separated from a square prism. ASME Journal of Fluids Engineering 113, 183-189.

Sreenivasan, K.R., Strykowski, P.J., 1990. On the formation and suppression of vortex shedding at low Reynolds number. Journal of Fluid Mechanics 218, 71-108.

Thiria, B., Wesfreid, J.E., 2006. Stability properties of forced wakes. Journal of Fluid Mechanics 579, 137-161.

Wu, Y.T., 1978. Cavity and wake flows. Annual Review of Fluid Mechanics 4, 243-284.

Zdravkovich, M.M., 1987. The effects of interference between circular cylinders in cross flow. Journal of Fluids and Structures 1, 239-261.

Zielinska, B., Wesfreid, J.E., 1995. On the spatial structure of global modes in wake flow. Physics of Fluids 7, $1418-1424$. 